\title{
1 Auxin is not asymmetrically distributed in initiating Arabidopsis leaves
}

2 Neha Bhatia $^{1}$ and Marcus G. Heisler ${ }^{1^{*}}$

\section{$4 \quad$ Affiliations}

$5 \quad{ }^{1}$ School of Life and Environmental Sciences, University of Sydney, NSW, Australia

6 *Author for correspondence: Marcus G Heisler (marcus.heisler@sydney.edu.au)

\section{Abstract}

It has been proposed that asymmetric auxin levels within initiating leaves help establish leaf polarity, based in part on observations of the DII auxin sensor. Here we show that the mDII control sensor also exhibits an asymmetry and that according to the ratio-metric auxin sensor R2D2, no obvious asymmetry in auxin exists. Together with other recent findings, our results argue against the importance of auxin asymmetry in establishing leaf polarity.

\section{Results and Discussion}

The leaves of seed plants are usually flat with distinct cell types making up their dorsal (upper) and ventral (lower) tissues. A fundamental question in plant development is how this dorsal-ventral polarity is first specified. Studies based on wounding experiments have suggested the presence of an inductive signal originating from the meristem that promotes dorsal identity in the adaxial (adjacent to the meristem) tissues of the leaf primordium ${ }^{1,2}$. However more recently, it was found that exogenous application of the plant hormone auxin to tomato leaf primordia resulted in the formation of radialized leaves that appeared ventrilized. Hence, relatively high levels of auxin are proposed to promote ventral or inhibit dorsal leaf cell fate ${ }^{3}$. Extending these conclusions, it was also proposed that an asymmetry in the auxin distribution across the leaf adaxial-abaxial axis at leaf initiation acts to help specify leaf polarity during regular development ${ }^{3}$. A critical piece of evidence supporting this latter proposal is that an auxin sensor, the $\mathrm{DII}^{4,5}$ indicates low levels of auxin in adaxial leaf tissues compared to abaxial tissues at leaf initiation ${ }^{3}$. Hence asymmetries

30 in auxin concentrations between the adaxial and abaxial leaf tissues, as a result of 31 PIN1 mediated auxin transport, are proposed to help establish leaf polarity ${ }^{3}$. Building on this conclusion, a more recent study proposed that low levels of auxin in adaxial 
tissues are necessary to restrict the expression of the WOX1 and PRS genes to the middle domain, since auxin promotes their expression ${ }^{6}$. Finally, the reported asymmetry in auxin has also been linked to asymmetries in the mechanical properties of leaf tissues and their morphogenesis ${ }^{7}$.

Despite these studies, reason to reassess the role of any asymmetry in auxin in specifying leaf polarity has arisen due to the recent finding that the expression patterns of genes involved in leaf dorsoventrality are already patterned in meristem tissues where leaves originate, with little change occurring to their expression during organ initiation. Hence leaf polarity appears pre-patterned ${ }^{8}$. Furthermore, rather than compromise dorsal cell fate, auxin was found to promote dorsal cell fate by maintaining Class III HD-ZIP expression and repressing KAN1 expression in the adaxial cells of organ primordia ${ }^{8}$, which is the opposite of what would be expected according to the auxin asymmetry hypothesis. In addition, Caggiano et al. (2017) also found that exogenous auxin application to young leaf primordia did not influence the spatial pattern of WOX1 and PRS, only the intensity of expression ${ }^{8}$. This also conflicts with the proposal that low levels of auxin are required in adaxial leaf tissues to prevent ectopic WOX1 and PRS expression as previously proposed ${ }^{6}$.

Given the concerns listed above, we decided to examine the distribution of auxin during leaf initiation using not only by the DII sensor assessed in previous studies but also, by looking more closely at the expression pattern of the non-auxin degradable mDII auxin sensor control ${ }^{4}$ as well as the ratiometric R2D2 auxin sensor ${ }^{9}$. We initially focused on the first two leaves three and four days after stratification (DAS), when these leaves are initiating as well as a day later as they begin to elongate and included the PIN1-GFP marker in our analysis to correlate auxin levels with PIN1 expression and polarity. At 3DAS, the expression patterns of REV and KAN1 are already polar within such primordia, although the FIL expression domain at this stage of development is still being refined ${ }^{8}$. Also at this stage, PIN1 is polarized towards the distal tip of leaf primordia but has reversed polarity away from the primordia, towards the meristem, in cells adjacent to the primordia on the adaxial side. According to the ratio-metric auxin sensor R2D2, auxin concentrations were relatively low in adaxial cells of the primordia but also low in abaxial and lateral regions proximally (Fig1-a and d; Fig.S1). High levels of auxin were only found in more distal regions towards 
the tip of the primordia, matching the overall pattern of signal from PIN1-GFP. No obvious asymmetry in signal between the adaxial and abaxial sides of the primordia was observed. This same overall pattern of signal was found in 16 out of 18 leaves that were examined. At 4DAS, the auxin distribution according to the R2D2 sensor was more uniform although higher levels of auxin appeared associated with the vasculature, again correlating with PIN1 expression ( $n=12 / 12$ leaves) (Fig. 1 g and j).

As our results using the R2D2 auxin sensor indicate a different auxin distribution compared to that reported previously using the DII marker ${ }^{3}$, we next re-examined the pattern of DII auxin sensor expression at the same developmental stages. In contrast to the R2D2 pattern, the DII pattern showed an asymmetry of expression in leaf primordia at $3 \mathrm{DAS}$, indicating relatively low auxin levels in adaxial primordium cells, as found previously. DII signal appeared strongest in the adaxial epidermis but was also stronger in the adaxial sub-epidermal cell layer compared to abaxial epidermal and sub-epidermal cell layers (Fig.1- b, e, h and Fig. S2) (n=18/18 leaves). One day later, although DII signal was still higher in the adaxial cells of the primordia, it started to show a relatively increased expression in the abaxial subepidermal layers as well compared to earlier stage ( $n=13 / 14$ leaves) (Fig1-k). Overall our results using the DII marker are similar to those obtained previously and consistent with the proposal that there are low levels of auxin in the adaxial regions of leaf primordia, in contrast to our results using the R2D2 sensor. Given this discrepancy, we next examined the expression of the mDII sensor which is driven by the same $35 \mathrm{~S}$ promoter as the DII sensor but is not auxin sensitive. Surprisingly we found that, like the DII results, expression of the mDII marker was also higher in the adaxial cells of the primordia ( $n=16 / 16$ leaves) (Fig.1-c,f,i and Fig.S3). The pattern appeared almost identical to the pattern found using the DII marker except that the mDII marker also showed high levels of expression in the shoot meristem whereas the DII sensor did not (compare Fig1-b,c; Fig S2 a-h and Fig S3 a-f ). The similarity of expression between DII and mDII was also apparent at 4DAS when the leaves had started to elongate (Fig.1-1) ( $n=14 / 14$ leaves). To verify the auxin sensitivity of the sensors used we imaged seedlings before and after treatment with 5mM NAA and found a strong decrease in DII expression compared to $\mathrm{mDII}$ and an increase in the ratio of VENUS compared to tdTomato signal for the R2D2 sensor, consistent with an increase in auxin levels (Figure S4). 
102 All together these results indicate that the asymmetry in expression found previously

103 for the DII auxin sensor in very young leaf primordia ${ }^{3}$ is not due to an asymmetry in

104 auxin levels but rather, likely due to differences in transcription driven by the $35 \mathrm{~S}$

105 promoter used to drive both DII and $\mathrm{mDII}$ in adaxial compared to abaxial leaf tissues.

106 We note that although a single section showing control expression of $\mathrm{mDII}$ in older

107 leaves was cited by Guan et al., $(2017)^{10}$, our results show that this information was

108 not adequate for assessing similarities and differences between DII and $\mathrm{mDII}$ at early

109 developmental stages. To check whether adaxial expression in leaf primordia is a

110 characteristic common to other reporters driven by the $35 \mathrm{~S}$ promoter, we also

111 examined the expression of 35S::H2B-mRFP1 and 35S::EGFP-LTI6b which had

112 previously been combined into the same plant line by crossing ${ }^{11}$. Surprisingly the

113 expression patterns for these markers were not only different to the mDII marker but

114 also different to each other with the H2B marker showing stronger expression in the

115 adaxial and abaxial epidermis and the LTI6b marker showing a more uniform pattern

116 (Fig. S5). These results reveal that even when the same promoter is utilized to drive

117 FP (fluorescent protein) expression, distinct differences in intensity patterns can occur

118 in planta. This may be due to differences in the position of T-DNA insertion in the

119 genome or differences in the surrounding DNA of the vector used. All together then,

120 our results highlight the importance of using ratio-metric sensors for in vivo

121 measurements since otherwise it is difficult to adequately control for differences in

122 signal intensity due to promoter activity or other confounding factors. Finally, while

123 the R2D2 marker utilizes a single transgene to express two distinct FPs from two

124 identical promoters ${ }^{9}$, a potentially improved approach to assess auxin levels in vivo

125 may be to utilize a single promoter to drive the auxin-dependent degradation domain

126 II from Aux/IAA proteins linked to a tandem fusion of fast and slowly folding FP

127 variants, such as VENUS and mCherry. Such an approach can enable a more direct

128 readout of protein degradation rates $^{12,13}$ and therefore potentially improve

129 measurements of relative auxin concentration.

131 While our findings are inconsistent with the proposal that asymmetries in the auxin

132 distribution influence leaf polarity ${ }^{3}$, pattern WOX/PRS gene expression ${ }^{6}$ or regulate

133 tissue mechanics ${ }^{7}$, they also leave other observations unexplained. In particular, to

134 understand the apparent ventrilization of leaf primordia in tomato in response to 
135 exogenous auxin ${ }^{3}$ will require further work in assessing how auxin distribution

136 patterns change in response to exogenous treatments and how auxin is distributed

137 during regular development in tomato, preferentially using a ratio-metric auxin sensor

138 such as R2D2.

139

140 Methods

141 Plant material and growth conditions.

142 Seeds of the plants expressing p35S::DII-VENUS and p35S::mDII-VENUS

143 transgenes (Columbia ecotype) were obtained from Dr. Teva Vernoux ${ }^{4}$. An

144 independent batch of seeds expressing p35S::mDII-VENUS transgenes (Columbia

145 ecotype) was also obtained from NASC (Arabidopsis stock center, NASC ID:

$146 \mathrm{~N} 799174)$ for analysis. R2D2 reporter line carrying pPIN1::PIN1GFP transgene

147 (Landsberg ecotype) has been described previously ${ }^{9,14}$. Seeds were germinated and

148 grown on GM medium ( $\mathrm{pH}-7$ with $1 \mathrm{M} \mathrm{KOH}$ ) containing 1\% sucrose, 1X Murashige

149 and Skoog salts (Sigma M5524), MES 2-(MN-morpholino)- ethane sulfonic acid

150 (Sigma M2933), 0.8 \% Bacto Agar (Difco), 1\% MS vitamins (Sigma M3900) in long

151 day conditions.

152

153 Confocal imaging and data analysis

154 Seedlings aged 3DAS (days after stratification) and 4DAS were dissected as 155 described previously ${ }^{14}$. Dissected seedlings were then oriented appropriately to obtain

156 a view of the young leaves either from above or from the side. Seedlings were imaged

157 live, on a Leica TCS-SP5 upright confocal laser scanning microscope with hybrid 158 detectors (HyDs) using a 25X water objective (N.A 0.95). VENUS was imaged using

159 argon laser (excitation wavelength $514 \mathrm{~nm}$ ) while tdTomato was imaged using a white

160 light laser (excitation wavelength $561 \mathrm{~nm}$ ). Z-stacks were acquired in a 512x512 pixel

161 format with section spacing of $1 \mu \mathrm{m}$ and line averaging 2.

162

163 Ratio-metric calculations for R2D2 auxin sensor were performed using ImageJ (FIJI,

$164 \mathrm{https}: / /$ fiji.sc) as described previously ${ }^{14}$. All the images were processed using IMARIS

1659.0 .0 (bit-plane). Optical sections (transverse or longitudinal) were reconstructed 166 using orthogonal slicer in IMARIS. 


\section{Auxin treatment}

170 Seedlings aged 3DAS were dissected imaged and treated with approximately $10 \mu \mathrm{L}$ of

$1715 \mathrm{mM}$ NAA (1-Napthaleneacetic acid) solution in water $(0.5 \mathrm{M}$ stock in $1 \mathrm{M} \mathrm{KOH})$ for

17260 minutes and imaged again with same settings as prior to treatment.

\section{Acknowledgements}

175 We thank Associate Professor Mary Byrne for critical feedback on the manuscript.

176 We thank Dr. Teva Vernoux for kindly providing the seeds of plants expressing $177 p 35 S:: D I I-V E N U S$ and $p 35 S:: m D I I-V E N U S$ transgenes. We also thank Professor Jim 178 Haseloff for kindly providing the seeds of plants expressing $p 35 S:: H 2 B-m R F P 1$ and p35S::EGFP-LTI6b transgenes.

\section{References}

1821 Sussex, I. M. Morphogenesis in Solanum tuberosum I: experimental 183 investigation of leaf dorsiventrality and orientation in the juvenile shoot. Phytomorphology 5, 286-300 (1955).

2 Reinhardt, D., Frenz, M., Mandel, T. \& Kuhlemeier, C. Microsurgical and laser ablation analysis of leaf positioning and dorsoventral patterning in tomato. Development 132, 15-26 (2005).

3 Qi, J. et al. Auxin depletion from leaf primordia contributes to organ patterning. Proc Natl Acad Sci $U$ S A 111, 18769-18774, doi:10.1073/pnas.1421878112 (2014).

4 Brunoud, G. et al. A novel sensor to map auxin response and distribution
at high spatio-temporal resolution. Nature 482, 103-106, doi:10.1038/nature10791 (2012).

5 Vernoux, T. et al. The auxin signalling network translates dynamic input into robust patterning at the shoot apex. Mol Syst Biol 7, 508, doi:10.1038/msb.2011.39 (2011).

6 Guan, C. et al. Spatial Auxin Signaling Controls Leaf Flattening in Arabidopsis. Curr Biol 27, 2940-2950 e2944, doi:10.1016/j.cub.2017.08.042 (2017).

7 Qi, J. et al. Mechanical regulation of organ asymmetry in leaves. Nat Plants

8 Caggiano, M. P. et al. Cell type boundaries organize plant development. eLife 6, doi:10.7554/eLife.27421 (2017).

9 Liao, C. Y. et al. Reporters for sensitive and quantitative measurement of auxin response. Nat Methods 12, 207-210, 202 p following 210, doi:10.1038/nmeth.3279 (2015).

10 Wang, Y. et al. The Stem Cell Niche in Leaf Axils Is Established by Auxin and Cytokinin in Arabidopsis. Plant Cell 26, 2055-2067, doi:10.1105/tpc.114.123083 (2014). 
11 Federici, F., Dupuy, L., Laplaze, L., Heisler, M. \& Haseloff, J. Integrated genetic and computation methods for in planta cytometry. Nature Methods 9, 483-U104, doi:10.1038/nmeth.1940 (2012).

12 Khmelinskii, A. et al. Tandem fluorescent protein timers for in vivo analysis of protein dynamics. Nat Biotechnol 30, 708-714, doi:10.1038/nbt.2281 (2012).

13 Barry, J. D., Dona, E., Gilmour, D. \& Huber, W. TimerQuant: a modelling approach to tandem fluorescent timer design and data interpretation for measuring protein turnover in embryos. Development 143, 174-179, doi:10.1242/dev.125971 (2016). 


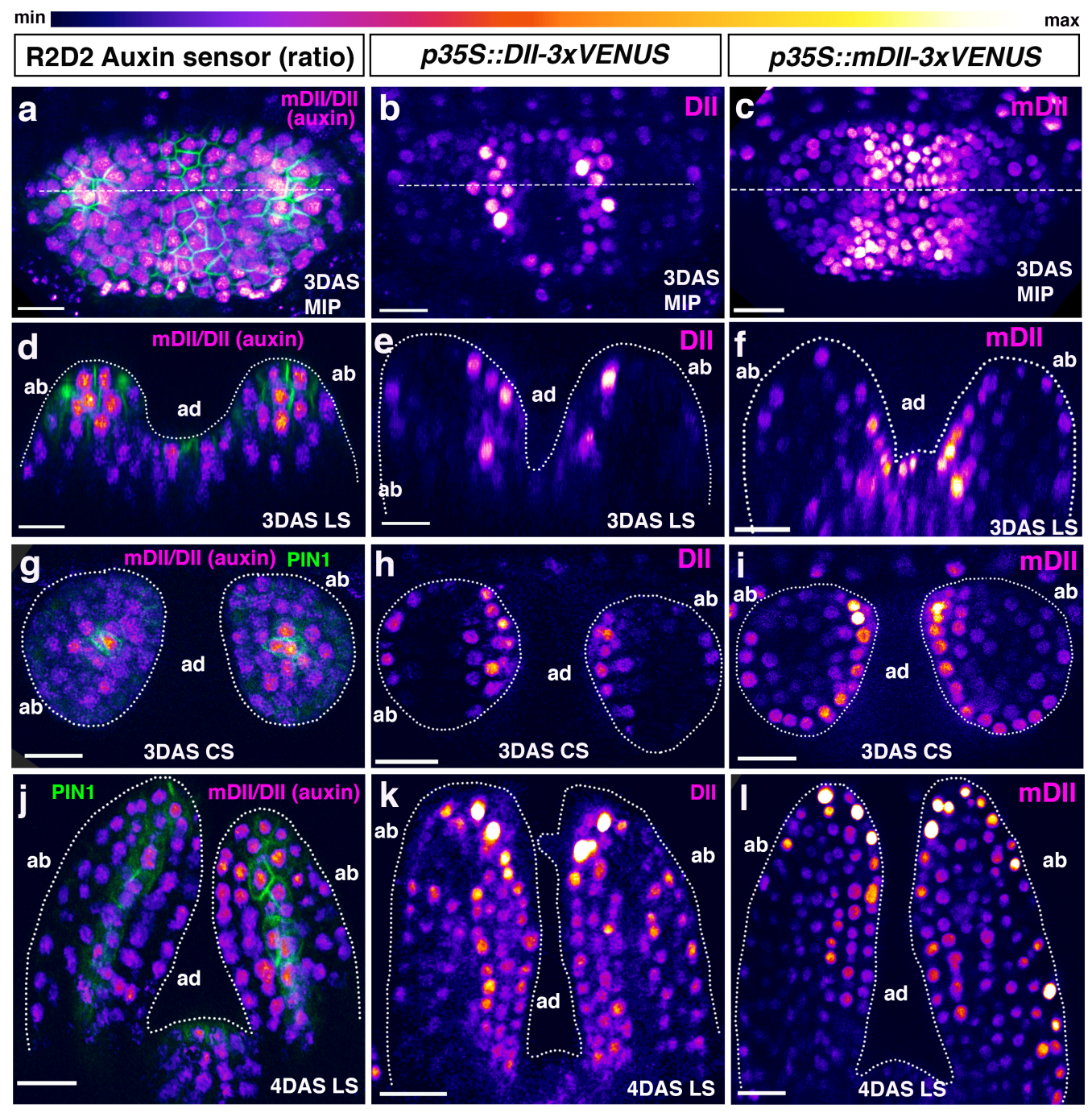

Figure 1 Predicted auxin distribution in young leaves as indicated by different 230 auxin sensors.

231 (a-c) Confocal projections of Arabidopsis seedlings aged 3DAS (days after 232 stratification) showing predicted auxin distribution based on the ratio-metric auxin 233 sensor R2D2 (magenta) along with PIN1-GFP (green) (a); expression pattern of 234 p35S::DII-VENUS (magenta) (b) and p35S::mutatedDII-VENUS (mDII, magenta) (c).

235 (d-f) Longitudinal reconstructed optical sections of (a-c), respectively, along the 236 dashed lines. (g-i) Representative examples of transverse reconstructed optical 237 sections of 3DAS Arabidopsis seedlings showing predicted auxin distribution as 238 indicated by the R2D2 sensor along with PIN1-GFP expression (g), DII-VENUS 239 expression (h) and mDII-VENUS expression (i). R2D2 auxin sensor indicates low but 240 symmetric auxin levels on adaxial and abaxial sides and relatively high auxin levels at 241 the distal tip and in the provasculature where PIN1 expression is also high (d, g). DII- 
242 VENUS is more strongly expressed adaxially indicating low auxin levels on the

243 adaxial side of the leaves relative to the abaxial side. However, mDII-VENUS also

244 shows high expression on the adaxial side of the leaf (compare e, h with $\mathrm{f}, \mathrm{i}$ ) and in

245 the shoot meristem. (j-l) Representative examples of longitudinal reconstructed

246 optical sections of 4DAS Arabidopsis seedlings showing predicted auxin distribution

247 by R2D2 sensor along with PIN1-GFP expression (green) (i), DII-VENUS expression

248 (j) and mDII-VENUS expression (k). At this stage, the R2D2 sensor indicates a more

249 uniform auxin distribution in the leaf but higher auxin levels in the vasculature where

250 PIN1-GFP expression is also high (j). DII-VENUS shows slightly high expression in

251 adaxial cells and an absence of expression in the vasculature $(\mathrm{k})$. mDII-VENUS

252 shows a similar pattern to DII but is also expressed in the vasculature (1). Scale bars

$25315 \mu \mathrm{m}(\mathrm{a}-\mathrm{i})$ and $20 \mu \mathrm{m}(\mathrm{j}-1)$. 


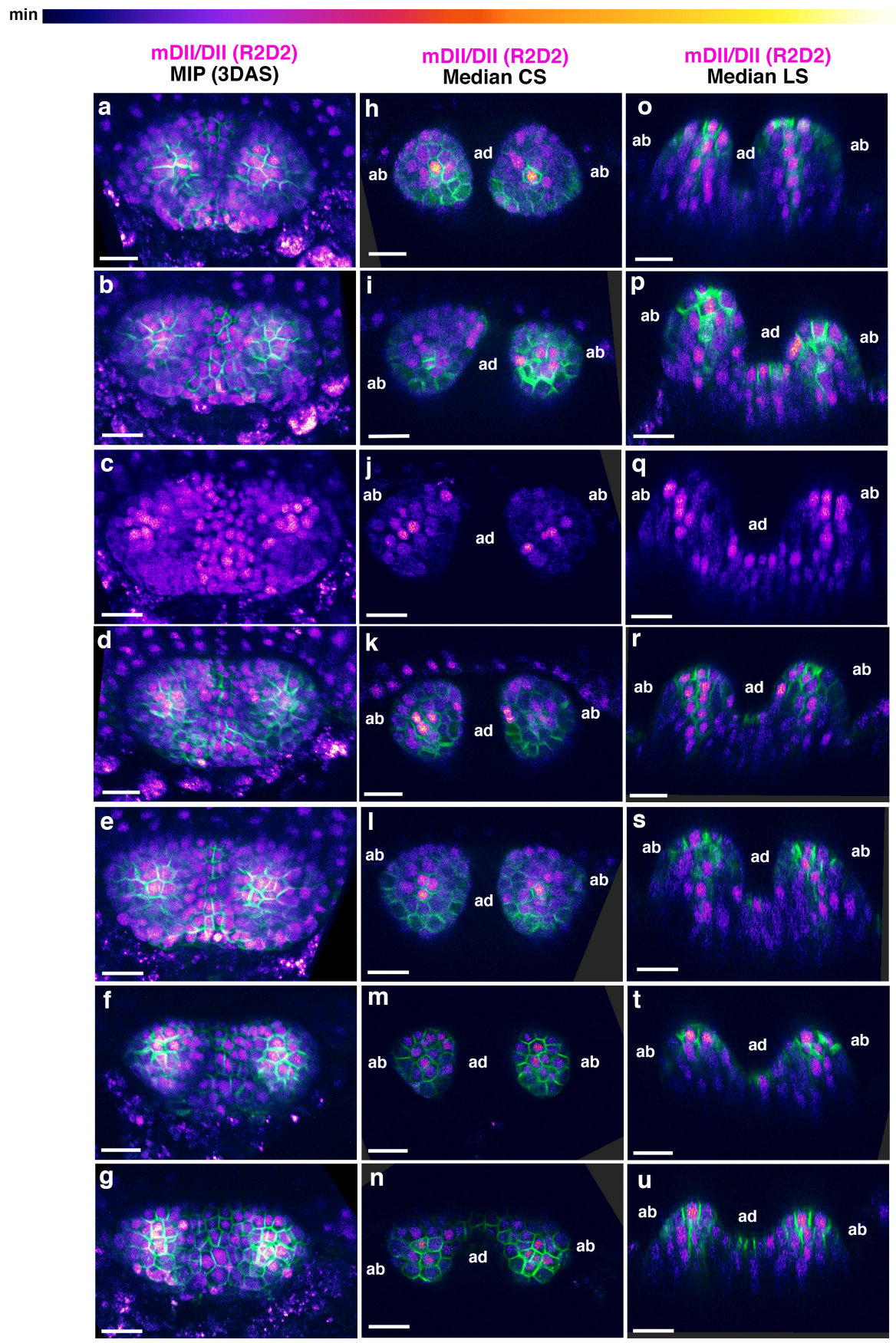

257 FigureS1 Additional examples of R2D2, ratio-based predictions of auxin 258 distribution in 3DAS old Arabidopsis seedlings. (a-g) Confocal projections of 259 Arabidopsis seedlings aged 3DAS (days after stratification) showing predicted auxin 260 distribution based on the ratio-metric auxin sensor R2D2 (magenta) along with PIN1261 GFP (green). (h-n) Median transverse optical sections of (a-g). (o-u) Median 262 longitudinal optical sections of (a-g). Scale bars $20 \mu \mathrm{m}(\mathrm{a}-\mathrm{u})$. 
bioRxiv preprint doi: https://doi.org/10.1101/284554; this version posted April 9, 2018. The copyright holder for this preprint (which was not

certified by peer review) is the author/funder, who has granted bioRxiv a license to display the preprint in perpetuity. It is made available under aCC-BY 4.0 International license.

265 Figure S2

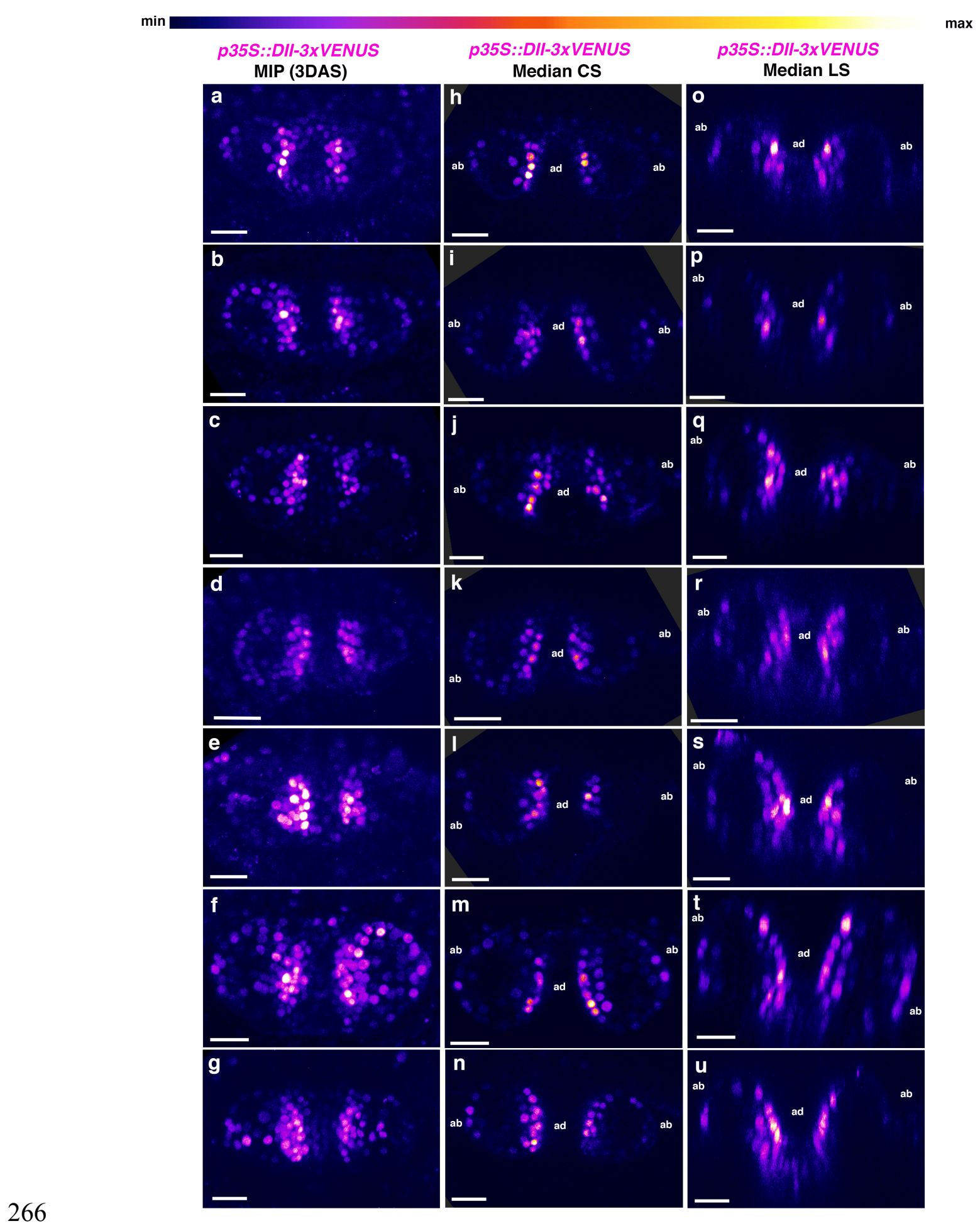

267 FigureS1 Additional examples p35S::DII-3xVENUS-N7 expression in 3DAS old

268 Arabidopsis seedlings. (a-g) Confocal projections of Arabidopsis seedlings aged

269 3DAS (days after stratification) showing expression pattern of $\mathrm{p} 35 \mathrm{~s}:$ :DII-3xVENUS- 
bioRxiv preprint doi: https://doi.org/10.1101/284554; this version posted April 9, 2018. The copyright holder for this preprint (which was not certified by peer review) is the author/funder, who has granted bioRxiv a license to display the preprint in perpetuity. It is made available under aCC-BY 4.0 International license.

270 N7 sensor (magenta). (h-n) Median transverse optical sections of (a-g). (o-u) Median

271 longitudinal optical sections of (a-g). Scale bars $20 \mu \mathrm{m}(\mathrm{a}-\mathrm{u})$. 


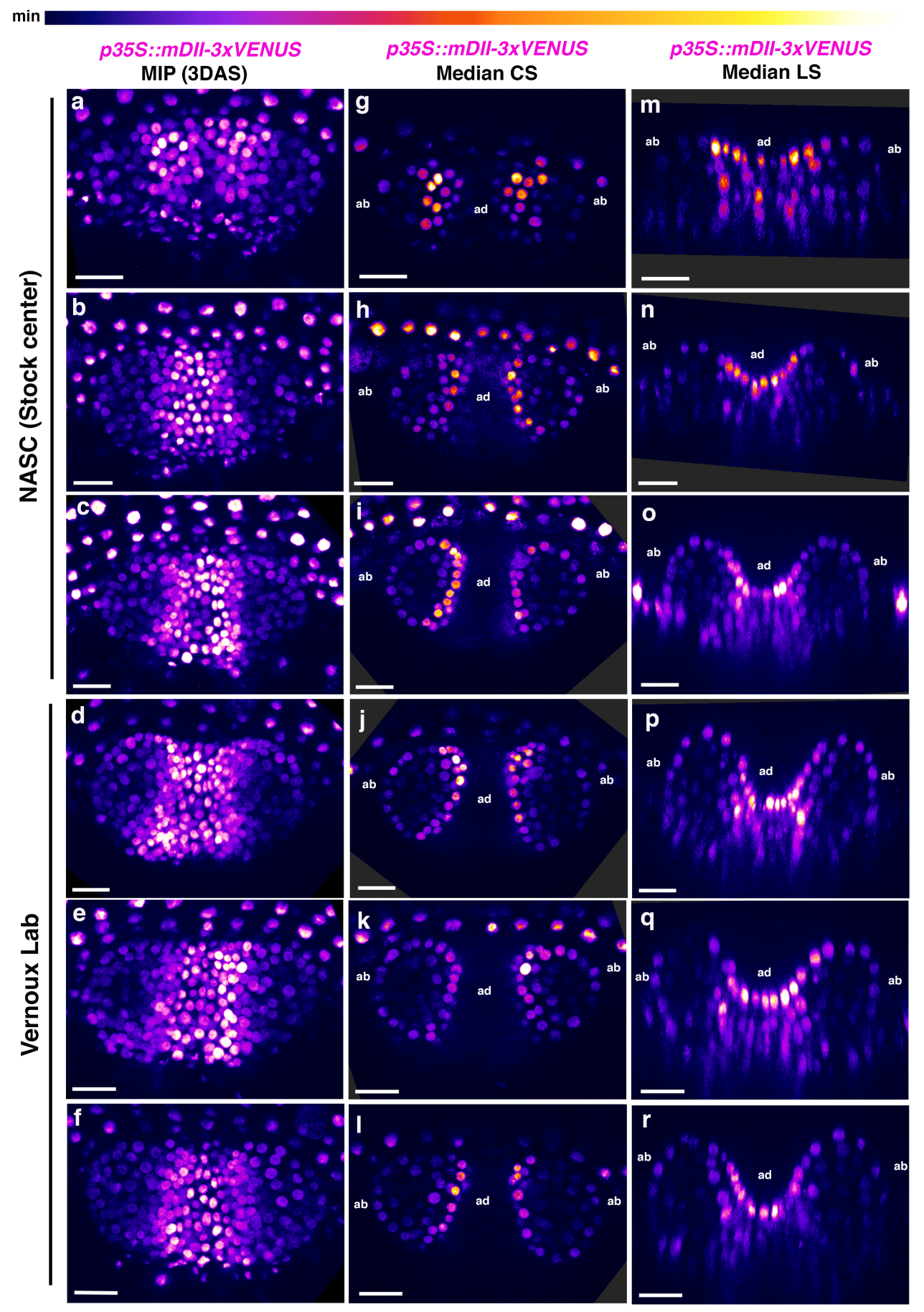

274 FigureS1 Additional examples of p35S::mDII-3xVENUS-N7 expression in 3DAS

275 old Arabidopsis seedlings. (a-f) Confocal projections of Arabidopsis seedlings aged

276 3DAS (days after stratification) showing expression pattern of p35s::mDII$2773 x$ VENUS-N7 sensor (magenta). Note that plants obtained from two different sources 
278 showed similar expression pattern. (h-l) Median transverse optical sections of (a-f).

279 (o-r) Median longitudinal optical sections of (a-g). Scale bars $20 \mu \mathrm{m}$ (a-r).

280

281

282

283

284

285

286

287

288

289

290

291

292

293

294

295

296

297

298

299

300

301

302

303

304

305

306

307

308

309

310 

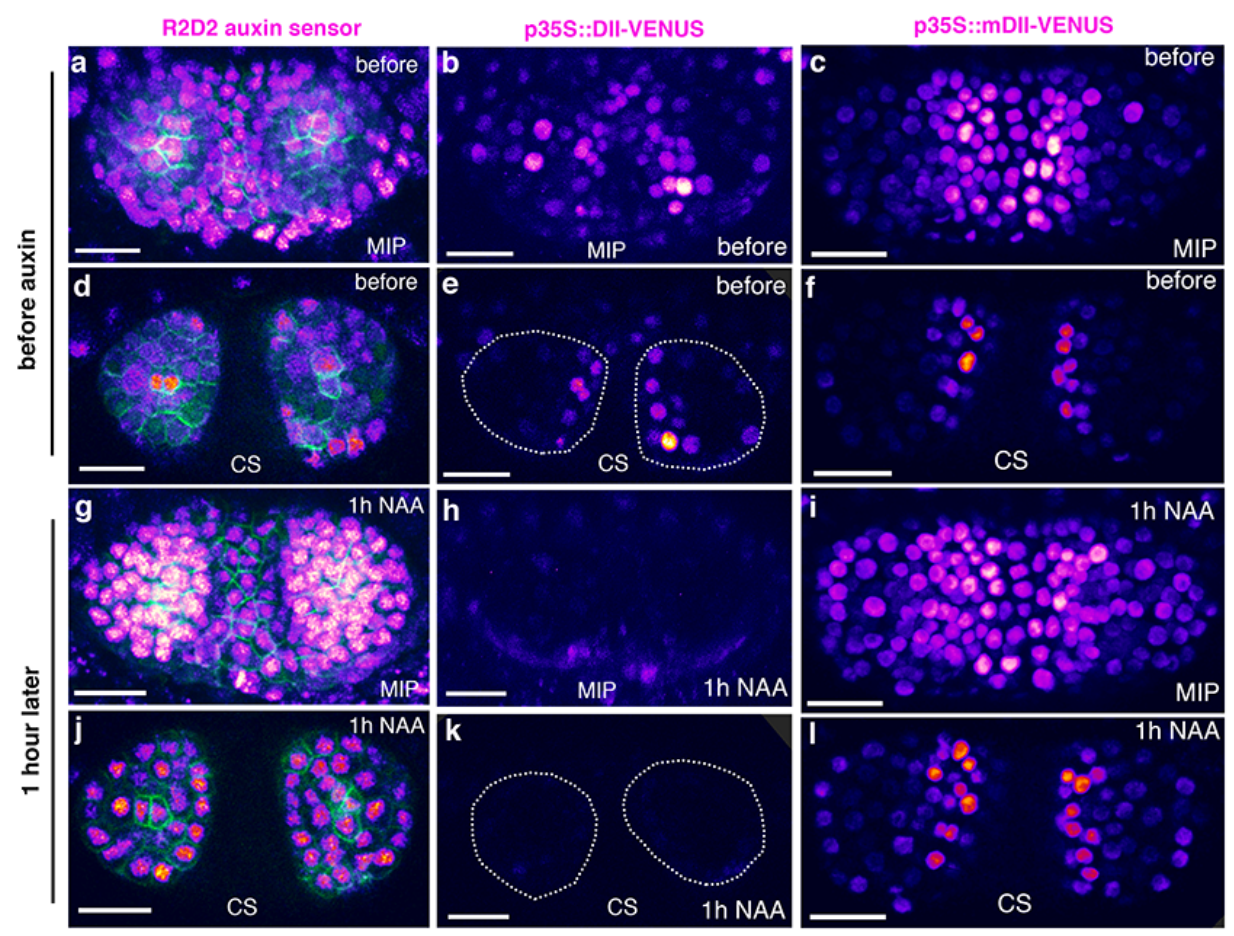

$\min$

\section{Figure S4 Response of different auxin sensors to external auxin application}

315 (a-f) Confocal projections (a-c) and transverse optical reconstructions (d-f) of 316 Arabidopsis seedlings, 3DAS, showing predicted auxin distribution based on R2D2

317 ratio-metric sensor (magenta) along with PIN1-GFP expression in green (a,d), DII-

318 VENUS sensor (magenta) (b,e) and mDII-VENUS sensor (magenta) (c, f) before 319 auxin application. (g-l) Confocal projections (g-i) and transverse optical 320 reconstructions (j-1) of Arabidopsis seedlings, 3DAS, showing predicted auxin 321 distribution based on R2D2 ratio-metric sensor (magenta) along with PIN1-GFP 322 expression in green (a,d), DII-VENUS sensor (magenta) (b,e) and mDII-VENUS 323 sensor (magenta) (c, f) 1hour after the application of 5mM NAA. Note, R2D2 sensor 324 indicates an increased and broadening of auxin levels after NAA application (compare $325 \mathrm{~d}$ and $\mathrm{j})(\mathrm{n}=3 / 3)$. Consistently, DII-VENUS shows an attenuated expression within 1 326 hour of auxin application (compare b,e with $h, j)(n=4 / 4)$. mDII-VENUS levels do not 327 decrease upon auxin application (compare c,f with $i, 1)(n=4 / 4)$. Scale bars $20 \mu \mathrm{m}(\mathrm{a}-1)$. 

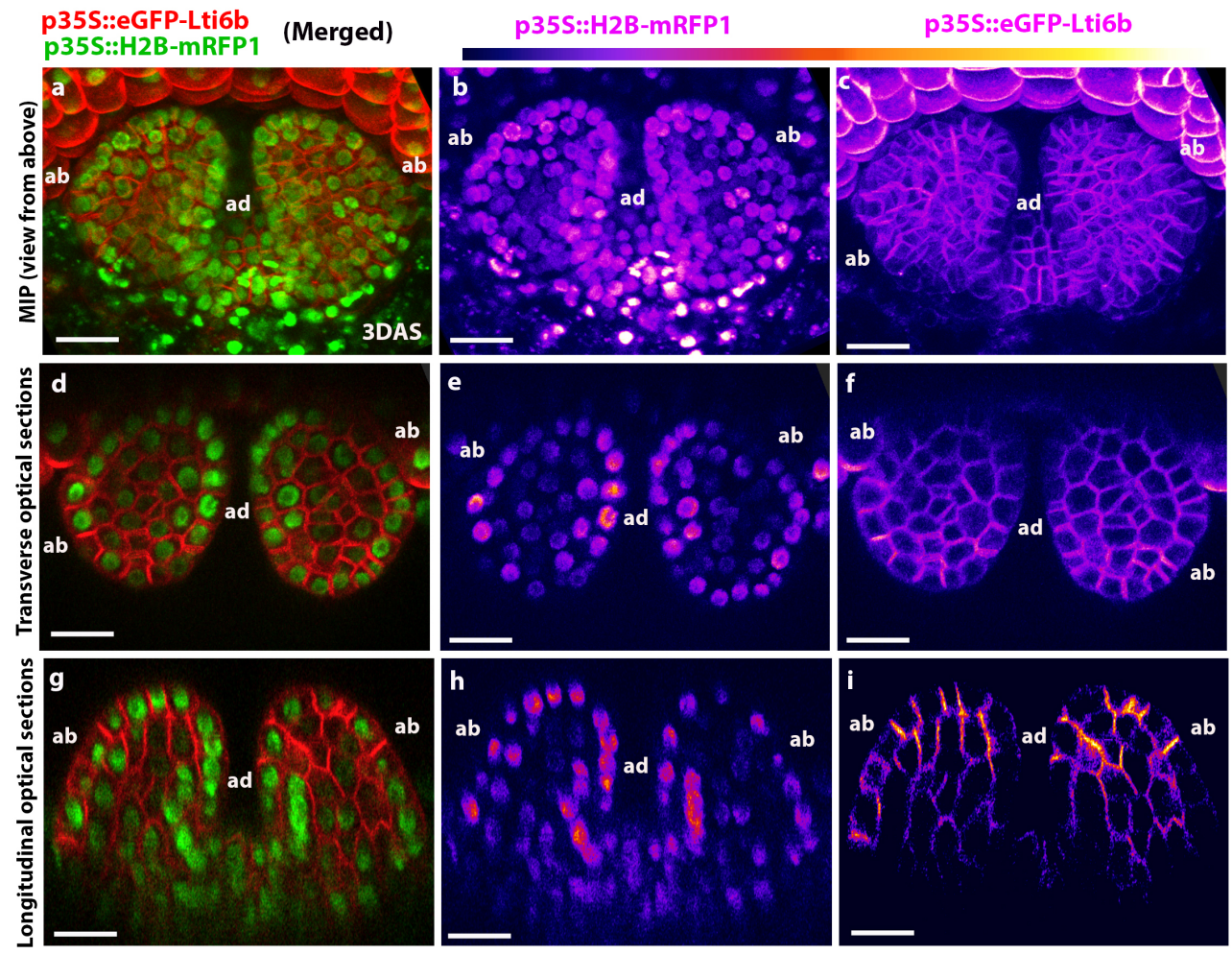

Figure S5 Different 35S promoter-driven reporter genes show different expression patterns in young leaves.

(a-c) Confocal projections of Arabidopsis seedlings aged 3DAS showing expression patterns of a p35S:eGFP-Lti6b (membrane marker, green) and p35S::H2B$m R F P 1$ (nuclear marker red) together (a), p35S::H2B-mRFP1 only (magenta) (b) and p35S:eGFP-Lti6b only (magenta) (c). (d-f) Transverse optical reconstructions of (a-c) respectively; merged (d), p35S::H2B-mRFP1 only (magenta) (e) and p35S:eGFPLti6b only (magenta) (i). Note $p 35 S:: H 2 B-m R F P 1$ shows similarly high levels of expression in both adaxial (ad) as well as abaxial (ab) epidermal cell layers and weaker expression in the middle regions (e and h). However, p35S:eGFP-Lti6b shows

346 no consistent asymmetry in expression throughout the leaf ( $\mathrm{f}$ and i). Scale bars $20 \mu \mathrm{m}$ 347 (a-i), $(\mathrm{n}=10$ leaves $)$. 